\title{
Variations in Stroke Patients' Muscle Activity during Head Rotation in Non-Paretic-Side Weight Bearing
}

\author{
Kwan-Sub Lee', Han-Seong Choe'2, Byung-Joo Lee ${ }^{3}$ \\ 'Department of Physical Therapy, Kang Hospital, Daegu; ${ }^{2}$ Department of Physical Therapy, The Graduated school, Catholic University of Daegu, \\ Daegu; ${ }^{3}$ Department of Emergency Medical Technology, Pohang College, Pohang, Korea
}

Purpose: This study aimed to determine the interaction among the neck, trunk, and lower extremities on the non-paretic side in head rotation along with non-paretic-side weight shifting of stroke patients. To compare stroke patients' ability to control posture through muscle activity variation related to pertubation during head rotation along with the non-paretic limb.

Methods: We tested 15 hemiplegic patients and 15 normal individuals. Each group's muscle activity was measured by electromyography in neutral head position and head rotation position. We compared each group's resu lt based on measured values in patients' non-paretic neck muscles, trunk muscles, and lower limbs muscles activation.

Results: The study showed that muscle activity increased in the sternocleidomastoid muscle (102.26\%,53.00\%), splenius capitis muscle $(97.93 \%, 54.93 \%)$, erector spinae muscle $(241.00 \%, 127.60 \%)$, external oblique abdominal muscle $(256.66 \%, 152.00 \%)$, and internal oblique abdominal muscle $(252.80 \%, 152.6 \%)$, peroneus longus muscle $(117.53 \%, 137.13 \%)$ and gastrocnemius muscle $(119.06 \%$, 137.20\%), while the results for the sternocleidomastoid muscle, splenius capitis muscle, erector spinae muscle, external oblique abdominal muscle, internal oblique abdominal muscle, peroneus longus muscle, and gastrocnemius muscle showed a statistically significant difference $(p<0.05)$.

Conclusion: It is hard for stroke patients to engage in normal movement control under suggested conditions because of the insufficient movement against gravity on the stroke patient's non-paretic side and impaired cooperative patterns. To solve these problems, patients need their bodies to improve through effective movement, resulting in advanced control of their effective and functional activity.

Keywords: Head movements, Stroke, Weight bearing

\section{INTRODUCTION}

Visual stimulation during daily activities when searching the surrounding environment requires balance in the standing position ${ }^{1,2}$ and horizontal visual movement is related to movements of other objects in the retina and results in controlled agitation. ${ }^{2}$ Movements through synchronization between the eyes and head are transferred into the neck and trunk, thereby enabling coordination in the motions of the four limbs, and include muscles in neck and head. ${ }^{3,4}$ Stroke patients experience changes in movements and stability through coordination of the head, trunk, and pelvis, which are required to move the body's weight, ${ }^{5,6}$ dominant use of the non-paret-

Received May 28, 2015 Revised Jun 15, 2015/Jun 17, 2015

Accepted Jun 18, 2015

Corresponding author Han-Seong Choe

E-mail khsc0224@gmail.com ic side due to a lack of balance control ability results in asymmetrical body weight support?

Postural control provides stability, as it gives a reference frame to move the head or four limbs selectively ${ }^{8}$ and control between connected segments is highly important for balance. Stroke patients have asymmetric body and muscle tone sclerosis, as well as sensory and motor nerve deficiencies. ${ }^{9}$ Damage to motor and sensory functions is a general symptom of stroke, representing a great obstacle to postural control and task-oriented activities. ${ }^{10}$ Due to the damage they have sustained, stroke patients have considerable difficulties in controlling balance in the standing position, along with motion, sensory, and cognitive problems; moreover, they face difficulties in

Copylight (C2015 The Korea Society of Physical Therapy

This is an Open Access article distribute under the terms of the Creative Commons Attribution Non-commercial License (Http:// creativecommons.org/license/by-nc/3.o.) which permits unrestricted non-commercial use, distribution, and reproduction in any medium, provided the original work is properly cited. 
moving the body weight to the non-paretic side during balance and gait performance. ${ }^{11}$ Dault et al. ${ }^{12}$ discovered that postural sway was found in the forehead of acute stroke patients and medial-lateral sway was related to asymmetry in chronic stroke patients. Furthermore, Anouk et al. ${ }^{13}$ showed a significant difference in postural control of voluntary head movements in the standing position between normal persons and stroke patients. In addition, Nicolas et al. ${ }^{14}$ and Geiger et al. ${ }^{15}$ studied sway characteristics due to asymmetric weight support and found that participants had more load on the non-paretic side.

As described above, most previous studies have focused on postural control on the paralyzed side against sway motion, as well as asymmetry in stroke patients. Thus, this study aimed to determine the interaction among the neck, trunk, and lower extremities on the non-paretic side in head rotation along with non-paretic-side weight shifting of stroke patients.

\section{METHODS}

\section{Study subjects}

The subjects of the present study were 15 patients with hemiplegia due to stroke who satisfied the study criteria, selected from patients who were admitted and treated with physical therapy in K Hospital in Daegu, Korea, and 15 normal persons whose ages were similar to those of the stroke patients. The subjects were informed about the test objectives and tasks and gave consent prior to the test. No significant differences in homogeneity and normality tests were found between the groups of subjects ( $p>0.05)$ (Table 1). The motor function and cognitive function of the subjects indicating Berg Balance Scale (BBS), Motor Assessment Scale (MAS), and Minimal Mental State Examination-Korean (MMSE-K) were as follows (Table 1).

Table 1. General characteristics of the subjects

\begin{tabular}{lccc}
\hline Division & Normal & Paretic & $p$ \\
\hline Male/female & $8 / 7$ & $10 / 5$ & 0.38 \\
Weight $(\mathrm{kg})$ & $62.80 \pm 6.44$ & $63.00 \pm 12.11$ & 0.61 \\
Mean age & $53.80 \pm 8.46$ & $55.70 \pm 6.56$ & 0.84 \\
Height $(\mathrm{cm})$ & $167.10 \pm 7.57$ & $164.90 \pm 4.15$ & 0.46 \\
BBS & $46.67 \pm 4.92$ & $38.33 \pm 6.78$ & \\
MAS & $41.87 \pm 2.72$ & $40.60 \pm 2.95$ & \\
MMSE-K & $27.00 \pm 1.60$ & $25.00 \pm 1.89$ & \\
\hline
\end{tabular}

Mean \pm SD: Mean Standard Deviation.

BBS, Berg Balance Scale; MAS, Motor Assessment Scale; MMSE-K, Minimal Mental State Examination-Korean.
The selection criteria for the subjects were as follows: (1) patients with hemiplegia due to cerebral infarction and cerebral hemorrhage; (2) patients who could walk at least $10 \mathrm{~m}$ independently without other assistance; (3) patients who had no orthopedic diseases of the pelvis and both lower extremities; (4) patients who could maintain standing balance with both feet together for at least 15 seconds; (5) patients who could understand and follow the researchers' instructions; (6) patients without vestibular disorder; and (7) patients without unilateral neglect.

\section{Measurement tools and procedures}

1) Surface electromyography

To measure muscle activity, surface electromyography (sEMG; MOTION LAB System Inc., LA, USA) was used. Data obtained as subjects rotated their heads were filtered and processed using WinDAQ software (DATAQ Instruments System Inc., OH, USA). A sample rate of EMG signals was set to $14,000 \mathrm{~Hz}$, and a $60 \mathrm{~Hz}$ notch filter was used to measure muscle activity. Root mean squares (RMSs) were calculated from the data obtained as described above and compared with each other.

\section{2) Muscle activity measurement region and method}

To determine muscle activity in the neck, trunk, and lower extremities during head rotation, recording electrodes were placed on muscles including the sternocleidomastoid (SCM), splenius cervicis (SP), external obliquus abdominis (EOB), internal obliquus abdominis (IOB), erector spinae (ES), peroneus longus (PL), and gastrocnemius (GCM). ${ }^{16,17}$ First, locations where recording electrodes should be placed were determined and electrode attachment regions were thoroughly cleansed with alcohol and shaved. For the muscle activities in the neck measured during head rotation while in a static standing position, In the trunk, lower extremities measured during maintained their heads in the neutral position during non-paretic-side weight shifting with both feet on the ground, a value in the reference voluntary contraction (RVC) test for each muscle was used.

Subjects rotated their heads to the sound actively and maintained the position for 5 seconds while the muscle activities in neck, trunk, and lower extremities were recorded. The subjects' arms were resting comfortably on the greater trochanter of the femur. Every motion was performed three times iteratively to measure muscle activity. 
The muscle's tension was measured and recorded as EMG signals for 5 seconds. The signals of the first and last seconds were removed and only the middle 3 seconds of the EMG signals were taken as data.

\section{Statistics}

The data acquired in this study were statistically processed using SPSS Version 14.0. The general characteristics of the study subjects were analyzed via descriptive statistics and an independent sample t-test was used to determine differences in muscle activities of the neck, trunk, and lower extremities between stroke patients and normal individuals. Furthermore, the Pearson correlation coefficient was used to determine correlation between muscle activities in the neck, trunk, and lower extremity on the non-paretic side of stroke patients. The statistical significance level, $\alpha$, was set as $\mathrm{p}<0.05$.

\section{RESULTS}

\section{Comparison of muscle activities between groups during head rotation}

To compare muscle activities in the neck, trunk, and lower extremities during head rotation, muscle activities in SCM, SP, EOB, IOB, ES, PL, and GCM were measured. Muscle activities on the non-paretic side were measured for the group of patients with hemiplegia, while muscle activities on the dominant side were measured for the normal group and their \%RVC values were compared.

\section{1) Comparison of muscle activities in the neck between groups}

The normal group showed increases in muscle activities in the SCM and SP of $102.26 \%$ and $97.93 \%$, while the hemiplegia group exhibit increases of 53.00\% and 54.93\%, and there were statistically significant differences in both the SCM and SP compared to the normal

Table 2. The comparison of neck, trunk, lower limb muscle activation in each groups

(\%/unit)

\begin{tabular}{lcccc}
\hline & Normal $(\mathrm{N}=15)$ & Paretic $(\mathrm{N}=15)$ & $\mathrm{t}$ & $\mathrm{p}$ \\
\hline SCM & $102.26 \pm 35.13$ & $53.00 \pm 25.01$ & -4.43 & $<0.001^{*}$ \\
SP & $97.93 \pm 24.47$ & $54.93 \pm 24.39$ & -4.82 & $<0.001^{*}$ \\
ES & $241.00 \pm 129.28$ & $127.60 \pm 41.21$ & -3.26 & $<0.001^{*}$ \\
EOB & $256.66 \pm 144.98$ & $152.00 \pm 62.37$ & -2.57 & $0.02{ }^{*}$ \\
IOB & $252.80 \pm 49.77$ & $152.60 \pm 64.15$ & -4.78 & $<0.001^{*}$ \\
PL & $117.53 \pm 13.23$ & $137.13 \pm 17.93$ & 3.41 & $<0.001^{*}$ \\
GCM & $119.06 \pm 10.10$ & $137.20 \pm 18.61$ & 3.32 & $<0.001^{*}$
\end{tabular}

SCM, Sternoclaviculomastoid; SP, Splenius; ES, Elector spinae; EOB, External oblique; $\mathrm{OB}$, Internal oblique; PL, Peroneus longus; GCM, gastrocnemius. ${ }^{*} \mathrm{p}<0.05$. group $(\mathrm{p}<0.05)($ Table 2$)$.

\section{2) Comparison of muscle activities in the trunk between groups}

The normal group demonstrated increased muscle activities in the ES, EOB, and IOB of $241 \%, 256.66 \%$, and $252.80 \%$, while the hemiplegia group had increases of $127.6 \%, 152 \%$ and $152.6 \%$; statistically significant differences compared to the normal group were seen in all of these muscles $(\mathrm{p}<0.05)($ Table 2$)$.

\section{3) Comparison of muscle activities in the lower extremities between} groups

The normal group exhibited increases in muscle activities in the PL and GCM of $117.53 \%$ and $119.06 \%$, while the hemiplegia group showed increases of $137.13 \%$ and $137.20 \%$. The differences between the hemiplegia group and normal group were statistically significant $(\mathrm{p}<0.05)($ Table 2$)$.

\section{Comparison of the correlation with muscle activities on the non-paretic side of hemiplegic patients}

During the muscle activity measurement using EMG, muscle activities on the non-paretic side of hemiplegic patients were measured to compare correlations with muscle activities in the neck, trunk, and lower extremities. The results showed that SCM and ES had a statistically significant correlation of 0.46 , while SP and ES had a statistically significant correlation of -0.47 . They also showed that the GCM and ES in the trunk and GCM and EOB had statistically significant correlations of 0.52 and 0.59 , respectively $(\mathrm{p}<0.05)$ (Table 3 ).

\section{DISCUSSION}

This study aimed to determine variations in stroke patients' muscle

Table 3. The comparison of correlation between muscle activation in paretic group

\begin{tabular}{lccccccc}
\hline \multicolumn{7}{c}{ Paretic group } \\
\cline { 2 - 8 } & SCM & SP & ES & EOB & IOB & PL & GCM \\
\hline SCM & 1 & 0.25 & $0.46 *$ & 0.14 & -0.06 & 0.09 & -0.17 \\
SP & & 1 & $-0.47 *$ & 0.22 & 0.23 & 0.25 & -0.24 \\
ES & & 1 & 0.37 & -0.43 & -0.01 & $0.52 *$ \\
EOB & & & 1 & -0.07 & -0.05 & $0.59 *$ \\
IOB & & & & & 1 & -0.33 & -0.45 \\
PL & & & & & & 1 & 0.28 \\
GCM & & & & & & & \\
\hline
\end{tabular}


activity in the neck, trunk, and lower extremities during head rotation while non-paretic-side weight shifting. We assumed that stroke patients would have postural control problems not only on the paretic side, but also on the non-paretic side; thus, we aimed to compare postural control abilities that maintain body balance against sways caused by weight shifting through the non-paretic side of stroke patients.

In this study, muscle activities in the neck during head rotation while weight shifting exhibited a greater increase than head rotation in the standing position. For example, the SCM in the neck demonstrated increases in muscle activity of $102.26 \%$ in the normal group and $53.00 \%$ in the hemiplegic group, while the SP showed increases in muscle activity of $97.93 \%$ in the normal group and $54.93 \%$ in the hemiplegic group. This result indicated that although the hemiplegic group had lower muscle activity than the normal group, the role of their neck muscles had changed, where they had become stabilizer muscles to increase resistance against sway. Furthermore, this result indicated that muscle activity around the neck to support the head during daily activities in the standing position compensated for the instability of body balance by means of increases in muscle activity and co-contraction in neck. This result is consistent with a study by Alessander et al. ${ }^{16}$ who proposed that although it was difficult to predict the direction and extent of head sway in medial-lateral sway, it was clear that stiffness of the neck increased in resistance against sways.

Brown et al. ${ }^{18}$ reported the importance of coordination between trunk muscles during sudden trunk sways, while Kim et al. ${ }^{19}$ reported that the trunk stability of stroke patients had a positive effect on subjects' dynamic balance ability. The present study indicated increased muscle activities in the ES, EOB, and IOB during head rotation of $241.66 \%, 256.66 \%$, and $252.80 \%$ in the normal group and $127.60 \%, 152.00 \%$, and $152.60 \%$ in the hemiplegic group. Through the above results, increased muscle activities in rotation muscles in normal and hemiplegic groups during head rotation helped to stabilize participants' trunks. This result was similar to those of a study by Duarte ${ }^{20}$ that reported unstable trunk control in stroke patients, as well as a study result by Roby-Brami et al. ${ }^{21}$ claiming that inefficient postural movements were caused by the limited range of motion of joints, as well as loss of appropriate coordination in trunk. However, in this study, muscle activities in the trunk due to head rotation in the stroke group were lower than those in normal group, and the reason for this seemed to be the promotion of inefficient muscle activities on the non-paretic side caused by the flexion pattern of trunk and biomechanical characteristics of the anterior pelvic tilt, which are commonly observed in stroke patients, although they were not measured in this study.

In this study, muscle activities on the medial side of the PL and GCM in the lower extremities were measured to determine the control abilities of internal and external muscles in the ankle joints during weight shifting to the non-paretic side. The results showed that the muscle activities during head rotation increased by $117.53 \%$ and $119.06 \%$ in the normal group, while those in the hemiplegia group increased by $137.13 \%$ and $137.20 \%$, respectively. Internal and external muscle activities in the ankle joints were higher in the hemiplegia group due to weakening of the pelvic mechanism than in the normal group, and this result indicated a trend of dominant use of the flexor and adductor in hip joints due to a reduction in control of tonic muscles required for postural control and an attempt to control the lower extremities via an inefficient approach that was dependent on biomechanical stability. This result is consistent that of Nicolas et al. ${ }^{14}$ who found that increased movements in the center of pressure (COP) mainly found along the medial and lateral axes could be seen as weakening the pelvis mechanism and controlled by internal and external muscles in the ankle joints.

The hemiplegic patients exhibited decreases in postural control muscles due to problems in balance and coordination, as well as asymmetric body support. This study aimed to determine the body control ability of hemiplegic patients against gravity through interaction of muscles in the head, trunk, and lower extremities during weight shifting to the non-paretic side. In terms of the relationship between muscles of the neck and trunk, the study showed that the SP and IOB had a statistically significant correlation $(\mathrm{p}<0.05)$, and the relationships between muscles of the neck and lower extremities showed that the SP and PL, as well as the SP and GCM, had statistically significant correlations $(\mathrm{p}<0.05)$. In the trunk, the ES and IOB, as well as the IOB and EOB, showed statistically significant correlations with each other $(\mathrm{p}<0.05)$. The above results are consistent with those of a study by Shumway-Cook et al. ${ }^{22}$ in which instability was compensated for by minimizing motions of the head and trunk, as well as those of a study by Roby-Brami et al. ${ }^{21}$ who showed that compensating postural control of muscles in the lower extremities and trunk can be expected due to sway applied directly to the 
head. Accordingly, the use of ankle joints-which are related to vertical stability in a static standing posture-incurs increases in lower extremity sensory inputs and muscle activities on the non-paretic side due to the reduction in muscle activities in the trunk during body sway, thereby representing a method that can be used to achieve postural control easily in standing position.

In this study, stroke patients had various postural control problems in the neck, trunk, and lower extremities on the non-paretic side due to muscle strength weakness, lack of body schema, and visual compensation. Moreover, stroke patients induced co-contraction by increasing muscle activities in the neck during sway and tended to compensate for postural instability through limited movements of the head by means postural stabilizing muscle rather than dynamic muscle function. Furthermore, appropriate coordination abilities on the non-paretic sides of stroke patients were reduced, and their postural control in the neck, trunk, and lower extremities during head rotation was different compared to that of the normal group.

The limitations of this study are as follows: The study result cannot be generalized to all stroke patients due to the small number of subjects. Moreover, comparison of muscle activities and changes in motion analysis between the paretic and non-paretic sides during weight shifting were not measured. These limitations will be overcome in future studies.

\section{ACKNOWLEDGEMENTS}

This research was supported by study year of Pohang college, 2014.

\section{REFERENCES}

1. Bronstein AM, Buckwell D. Automatic control of postural sway by visual motion parallax. Exp Brain Res. 1997;113:243-8.

2. Guerraz M, Sakellari V, Burchill P, et al. Influence of motion parallax in the control of spontaneous body sway. Exp Brain Res. 2000;131:244-52.

3. Nashner LM. Adaptation of human movement to altered environments. TINS. 1982;358-61.

4. Dietz V. Human neuronal control of automatic functional movements: interaction between central programs and afferent input. Physiol Rev. 1992;72:33-69.
5. Danielsson A, Willen C, Sunnerhagen KS. Measurement of energy cost by the physiological cost index in walking after stroke. Arch Phys Med Rehabili. 2007;88:1298-303.

6. Lamontagne A ,Serres SJ, Fung J, et al. Stroke affects the coordination and stabilization of head, thorax and pelvis during voluntary horizontal head motions preformed in walking. Clinical Neurophysiology. 2005; 116:101-11.

7. Bente E, Bassoe G. The Bobath concept in adult neurology. Thieme, Stuttgart, New York. 2008;109.

8. Keshner EA, Dhaher Y. Characterizing Head Motion in 3Planes during Combined Visual and Base of Support Disturbances in Healthy and Visually Sensitive Subjects. Gait Posture. 2008;28(1):127-34.

9. Shim HB, Cho HY, Choi WH. Effects of the trunk stabilization exercise on muscle activity in lumbar region and balance in the patients with hemiplegia. J Kor Phys Ther. 2014;26(1):33-40.

10. Song BK. Effect of Somatosensory Stimulation on Upper Limb in Sensory, Hand Function, Postural Control and ADLs within Sensorimotor Deficits after Stroke. J Kor Phys Ther. 2012;24(5):291-9.

11. Kim JH. A study on the correlation between static, dynamic standing balance symmetry and walking function in stroke. J Kor Phys Ther. 2012;24(2):73-81.

12. Dault MC, De Haart M, Geurts AC, et al. Effects of visual center of pressure feedback on postural control in young and elderly healthy adults and in stroke patients. Hum Mov Sci. 2003;22(3):221-36.

13. Anouk L, Nicole P, Joyce F. Postural adjustments to voluntary head motions during standing. Clinical Biomechanics. 2003;18:832-42.

14. Nicolas G, Patrice R, Anne-Sophie G, et al. Contribution of Each Lower Limb to Upright Standing in Stroke Patients. Stroke. 2008;39:1793-9.

15. Geiger RA, Allen JB, O’Keefe J, et al. Balance and mobility following stroke: effects of physical theraphy interventions with and without biofeedback/forceplate training. Phys Ther. 2001;81:995-1005.

16. Alessander DDS, Adriana M, Degani, et al. Anticipatory Control of Head Posture. Clin Neurophysiol. 2007;118(8):1802-14.

17. Ingrid B.M Van Der Fits, Mijna HA. The development of postural response patterns during reaching in healthy infants. Neuroscience \& Biobehavioral Reviews. 1998;22(4):521-6.

18. Brown SHM, Vera-Garcia FJ, McGill SM. Effects of abdominal muscle coactivation on the externally pre-loaded trunk. variations in motor control and its effect on spine stability. Spine. 2006;31(13):387-93.

19. Kim GH, Choe HS, Lee HI, et al. The Effects of Scapular Stabilization Exercising on Dynamic Standing Balance in Stroke Patients. J Kor Phys Ther. 2014;2(1):15-20.

20. Duarte E, Marco E, Muniesa JM, et al. Trunk control test as a functional predictor in stroke patients. J Rehabil Med. 2002;34(6):267-72.

21. Roby-Brami A, Feydy A, Combeaud M, et al. and rec Motor compensation overy for reaching in stroke patients. Acta Neurologica Scandinavica. 2003;107(5):369-81.

22. Shumway-cook A, Horak FB. Rehabilitation strategies for patients with vestibular deficits. Neurologic Clinics. 1990;8:441-57. 